\title{
Activation of the KEAP1-NRF2-ARE signaling pathway reduces oxidative stress in Hep2 cells
}

\author{
CHANGJIANG LI ${ }^{1}$, LEI CHENG ${ }^{1}$, HAITAO WU ${ }^{1}$, PEIJIE HE ${ }^{1}$, YANPING ZHANG ${ }^{2}$, \\ YUE YANG $^{1}$, JIAN CHEN ${ }^{1}$ and MIN CHEN ${ }^{1}$ \\ ${ }^{1}$ Department of Otorhinolaryngology-Head and Neck Surgery, Shanghai Key Clinical Disciplines of Otorhinolaryngology, \\ Fudan University; ${ }^{2}$ Department of Central Laboratory, Eye, Ear, Nose and Throat Hospital, \\ Fudan University, Shanghai 200031, P.R. China
}

Received August 29, 2017; Accepted February 9, 2018

DOI: $10.3892 / \mathrm{mmr} .2018 .9288$

\begin{abstract}
At present there are no studies investigating the effects of the kelch-like ECH-associated protein 1 (KEAP1)-nuclear factor erythroid 2-related factor 2 (NRF2)-antioxidant response element (ARE) signaling pathway on Hep2 cell line. The present study aimed to investigate this topic through knockdown of the KEAP1 gene. A stable Hep2 cell line specifically silencing the human KEAP1 gene was initially constructed. Hydrogen peroxide $\left(\mathrm{H}_{2} \mathrm{O}_{2}\right)$ was added to the culture medium at various concentrations for various durations to interact with the short hairpin (sh)KEAP1-transfected Hep2 cells. Subsequently, the gene and protein expression levels of KEAP1, NRF2, NAD(P)H quinone oxidoreductase1 (NQO1) and heme oxygenase 1 (HO1) in experimental and control cells were measured by reverse transcription-quantitative polymerase chain reaction and western blotting, respectively. Furthermore, the viability and apoptotic rate of the shKEAP1-transfected Hep2 cells were detected by a Cell Counting-Kit 8 assay and flow cytometry, respectively. In the shKEAP1 Hep2 cell line, the mRNA and protein expression levels of NRF2, NQO1 and HO1 were markedly higher compared with the scramble control-transfected Hep2 and parent Hep2 cell lines. Immunofluorescence staining indicated that NRF2 was primarily located in the cytoplasm of scHep2 and parent Hep2 cell lines, but was present in the nuclei and cytoplasm of the shKEAP1 Hep2 cell line, where it translocates into the nuclei in response to $\mathrm{H}_{2} \mathrm{O}_{2}$. Following knockdown of the KEAP1 gene Hep2 cells, the apoptosis rates were 31.8 and $45.3 \%$ in scHep2 cells at 0.1 and $0.25 \mathrm{mmol} / 1$
\end{abstract}

Correspondence to: Dr Lei Cheng, Department of Otorhinolaryngology-Head and Neck Surgery, Shanghai Key Clinical Disciplines of Otorhinolaryngology, Fudan University, 83 Fenyang Road, Xuhui, Shanghai 200031, P.R. China

E-mail: tomorrowhot@163.com

Key words: anti-oxidative effect, kelch-like ECH-associated protein 1-nuclear factor erythroid 2-related factor 2-antioxidant response element signaling pathway, Hep2 cells, hydrogen peroxide
$\mathrm{H}_{2} \mathrm{O}_{2}$ respectively and 14.1 and $27.9 \%$ in shKEAP1 cells. The present study indicated that the KEAP1-NRF2-ARE signaling pathway may exhibit an antioxidative effect within Hep2 cells and may be used for clinical treatment of cancer.

\section{Introduction}

There is an increasing body of evidence demonstrating that oxidative stress may be associated with cancer. Oxidative stress triggers the modification of DNA bases, DNA fragmentation and strand breaks, which contribute to the development of numerous cancer types as well as various other diseases (1). Numerous studies concerning the association between oxidative stress and cancer have been previously conducted (2-4). When confronted with external oxidative damage, the body will have evolved numerous defense systems to remove the stimulus. The kelch-like ECH-associated protein 1 (KEAP1)/nuclear factor erythroid 2-related factor 2 (NRF2) system is one of the most important cell defense systems and survival pathways in vivo (5). NRF2 serves a core role in this pathway. NRF2 is anchored in the cytoplasm by KEAP1 in the resting state and translocates into the nucleus to activate the antioxidant response element (ARE) under oxidative stress conditions, which may lead to an increase in the expression of downstream antioxidative proteins, including $\mathrm{NAD}(\mathrm{P}) \mathrm{H}$ quinone oxidoreductase 1 (NQO1) and heme oxygenase 1 (HO1) (6). NQO1 and $\mathrm{HO} 1$ are regarded as inducible phaseIIdetoxifying enzymes. NQO1 is a flavoprotein that protects the body from oxidative damage via stabilization of the p53 tumor suppressor (7). HO1 catalyzes the initial and rate-limiting steps in heme catabolism and exhibits a protective effect by decreasing the intracellular pro-oxidant levels (8). However, it has been reported that as well as protecting normal cells from oxidative damage, NRF2 also protects tumor cells. This finding has been confirmed within numerous cell lines and tissues, including non-small cell lung carcinoma, pancreatic cancer and ovarian cancer (7,9-11). Selective knockdown of KEAP1 with small interfering (si)RNA was reported to promote the nuclear migration and expression of NRF2 and its downstream genes in human umbilical vein endothelial cells (12). Furthermore, research by Wakabayashi et al reported that $\mathrm{KEAP}^{-/}$mice are more likely to die postnatally due to malnutrition resulting 
from hyperkeratosis in the esophagus and forestomach; however, simultaneous ablation of NRF2 may reverse KEAP1 deficiency-associated phenotypes (13).

To the best of our knowledge, no previous studies concerning an association between the Hep2 cell line and the KEAP1/NRF2 signaling pathway have been reported. Therefore, in the present study, the effects of KEAP1 knockdown on NRF2 and its downstream elements were investigated using RNA interference (RNAi) to reveal the integrity of the KEAP1/NRF2 system and the effect on oxidative stress in the Hep2 cell line following the addition of hydrogen peroxide $\left(\mathrm{H}_{2} \mathrm{O}_{2}\right)$.

\section{Materials and methods}

Cell lines and cell culture. The Animal Ethics Committee of the Eye, Ear, Nose and Throat Hospital of Fudan University (Shanghai, China) reviewed and approved the study protocol. The Hep2 cell line employed in the present study was from our own laboratory (Laboratory Center, Eye, Ear, Nose and Throat Hospital of Fudan University, Shanghai, China). Cells were maintained in RPMI-1640 (Hyclone; GE Healthcare Life Sciences, Logan, UT, USA) with $10 \%$ fetal bovine serum (Gibco; Thermo Fisher Scientific, Inc., Waltham, MA, USA) and penicillin $(50 \mathrm{U} / \mathrm{ml})$-streptomycin $(50 \mu \mathrm{g} / \mathrm{ml})$ solution (Gibco; Thermo Fisher Scientific, Inc.). The cell line was incubated at $37^{\circ} \mathrm{C}$ in a humidified atmosphere of $95 \%$ air and $5 \% \mathrm{CO}_{2}$. The mixed cancer Hep2 cell line, which was originally considered to be of the laryngeal carcinoma type but was later reported to be contaminated with cervical carcinoma HeLa cells, was used as a cancer cell model in the current study (14-16).

Construction of lentivirus vectors. According to the human KEAP1 transcript in GenBank (https://www.ncbi. nlm.nih.gov/nuccore/; NM_203500), three target RNA interference sequences that silence the KEAP1 gene were identified. Lentiviral vectors expressing RNAi specific for the KEAP1 gene and a scrambled sequence encoding a green fluorescent protein (GFP) sequence were designed and constructed by Obio Technology Co., Ltd. (Shanghai, China). The following sequences were used: 5'-GCAAGGACTACC TGGTCAAGA-3' (shKEAP1), 5'-CGGGAGTACATCTAC ATGCAT-3' (shKEAP1-1), 5'-GTGGCGAATGATCACAGC AAT-3' (shKEAP1-2) and 5'-TTCTCCGAACGTGTCACGT-3' (scRNA). Pairs of complementary oligonucleotides with these sequences were synthesized, annealed and cloned into the lentiviral plasmid vector [pLKD-CMV-G\&PR-U6-short hairpin (sh)RNA] (Obio Technology, Ltd., Shanghai, China) using the AgeI and EcoRI enzymes (Takara Bio, Inc., Otsu, Japan). The recombinant plasmid vectors (32 $\mu \mathrm{g})$ containing shRNA were co-transfected into 293T cells along with the helper plasmids psPAX2 and pHCMV-VSV-G (Takara Bio, Inc.) using Lipofectamine ${ }^{\circledR} 2000$ (Invitrogen; Thermo Fisher Scientific, Inc.). Subsequently, the supernatants were collected and purified after $48 \mathrm{~h}$ of transfection and then titrated.

The constructed lentiviral vectors contained a green fluorescence gene that emits green fluorescence in response to excitation with blue light. This characteristic may be utilized to optimize the transfection conditions. Following the screening of a series of preliminary experiments using $2 \mu \mathrm{g} / \mathrm{ml}$ puromycin, the optimal transfection efficiency was determined. Briefly, for cell transfection, Hep2 cells in the exponential growth phase were collected, counted and seeded in 24-well tissue culture plates (Corning Incorporated, Corning, NY, USA) at a density of $2 \times 10^{4}$ cells/well to attain $30 \%$ confluence on the day of transfection. The lentivirus containing specific KEAP1 shRNA and the negative control lentivirus were applied to Hep2 cells with the multiplicity of infection of 10 or 20. In addition, polybrene was added into each well with a final concentration of $5 \mu \mathrm{g} / \mathrm{ml}$. At $12-20 \mathrm{~h}$ post-transfection, the medium was completely replaced and the cells were incubated at $37^{\circ} \mathrm{C}$ in a $5 \% \mathrm{CO}_{2}$ incubator for an additional $72 \mathrm{~h}$. The transfection rate was evaluated by counting GFP-positive cells under an inverted fluorescence microscope; silencing efficacy was verified using real-reverse transcription-quantitative polymerase chain reaction (RT-qPCR), western blotting and an immunofluorescence technique. Hep2 cells that exhibited the highest degree of KEAP1 silencing following transfection with lentivirus containing KEAP1-shRNA were selected for the establishment of a stable cell line in the presence of puromycin at a final concentration of $2 \mu \mathrm{g} / \mathrm{ml}$. The media containing puromycin were changed every 2-3 days, for 2 weeks. Parent Hep2 and scHep2 cells served as the controls.

Detection of apoptosis by flow cytometry. Cells were seeded in 6-well plates (Corning Incorporated) at a density of $4 \times 10^{5}$ cells/well and cultured for $24 \mathrm{~h}$. Subsequently, cells were treated with two different concentrations of $\mathrm{H}_{2} \mathrm{O}_{2}(0.1$ and $0.25 \mathrm{mmol} / \mathrm{l}$ ) for $24 \mathrm{~h}$ at $37^{\circ} \mathrm{C}$. The floating original medium was collected and the cells were digested with $0.25 \%$ trypsin without EDTA (Hyclone; GE Healthcare Life Sciences) at $37^{\circ} \mathrm{C}$ for $1 \mathrm{~min}$. Cell suspensions were centrifuged for $5 \mathrm{~min}$ at $1,000 \mathrm{x} \mathrm{g}$ at room temperature. Cells were resuspended and gently washed twice with ice-cold phosphate-buffered saline (PBS; pH 7.2-7.4; Hyclone; GE Healthcare Life Sciences). Finally, the cells were collected in $1.5 \mathrm{ml}$ Eppendorf tube. Cell density was adjusted to $1 \times 10^{6}$ cells/tube. According to the operating instructions of an Annexin V-allophycocyanin (APC)/7-aminoactinomycin D (7-AAD) double staining apoptosis detection kit (Nanjing KeyGen Biotech Co., Ltd., Nanjing, China), $500 \mu \mathrm{l}$ binding buffer was added to suspend cells. A total of $5 \mu \mathrm{l}$ Annexin V-APC and 7-AAD was subsequently added respectively for flow cytometry within 1 h using a Beckman MoFlo XDP flow cytometer with Summit 5.2 (Beckman Coulter, Inc., Brea, CA USA). Cells not treated with any $\mathrm{H}_{2} \mathrm{O}_{2}$ served as the controls.

Cell viability assay. shKEAP1 Hep2 and scHep2 cell lines in the logarithmic growth phase were trypsinized, counted, resuspended and plated at a density of $2 \times 10^{3}$ cells/well in a volume of $200 \mu \mathrm{l}$ RPMI-1640 medium per well in a 96-well plate in triplicate. After 6, 24, 48, 72 and $96 \mathrm{~h}$ incubation individually (data not shown), cell proliferation was measured using a Cell Counting Kit-8 (CCK-8; Dojindo Moleular Technologies Inc., Kumamoto, Japan) according to the manufacturer's protocols. The absorbance value was recorded at $450 \mathrm{~nm}$ using a microplate spectrophotometer. To quantify the ability of $\mathrm{H}_{2} \mathrm{O}_{2}$ to inhibit the shKEAP1 Hep2 cell line, a cell growth assay was also performed using CCK-8. Briefly, cells were seeded in a 
Table I. Sequences of reverse transcription-quantitative polymerase chain reaction oligonucleotide primers.

\begin{tabular}{lll}
\hline Gene & \multicolumn{1}{c}{ Forward sequence } & \multicolumn{1}{c}{ Reverse sequence } \\
\hline NRF2 & CGTCCCAGCAGGACATGGAT & AGCTCATACTCTTTCCGTCGC \\
KEAP1 & TCCCCTACAGCCAAGGTCC & TCAGTGGAGGCGTACATCAC \\
NQO1 & CCTTCCGGAGTAAGAAGGCAG & TCCAGGCGTTTCTTCCATCC \\
HO1 & ATGACACCAAGGACCAGAGC & GCATAAAGCCCTACAGCAACT \\
GAPDH & CAGGAGGCATTGCTGATGAT & GAAGGCTGGGGCTCATTT
\end{tabular}

NRF2, nuclear factor erythroid 2-related factor 2; KEAP1, kelch-like ECH-associated protein; NQO1, NAD(P)H quinone oxidoreductase 1; HO1, heme oxygenase 1 .

96-well tissue culture plate $\left(2 \times 10^{4} /\right.$ well $)$, incubated overnight at $37^{\circ} \mathrm{C}$ and subsequently treated with $0,0.25,0.5,0.75$ and $1.0 \mathrm{mmol} / \mathrm{l}$ of $\mathrm{H}_{2} \mathrm{O}_{2}$ for $24 \mathrm{~h}$. CCK-8 was subsequently added to each well to detect the inhibitory effect of $\mathrm{H}_{2} \mathrm{O}_{2}$ on the cells using a microplate spectrophotometer at a wavelength of $450 \mathrm{~nm}$.

Immunofluorescence staining. Cells in the exponential growth phase were digested with trypsin, resuspended and the cell density was adjusted to $1 \times 10^{6}$ cells $/ \mathrm{ml}$ with culture media. Prior to cell density adjustment, one glass coverslip (22x22 mm) was placed into each well of a 6 -well dish. A total of $5 \mu 1$ cell suspensions was seeded onto the glass coverslip in order to grow up to $30-50 \%$ confluence 24 h later. $\mathrm{H}_{2} \mathrm{O}_{2}$ was added into the wells at final concentrations of 0.25 and $0.5 \mathrm{mmol} / 1$ for 6 and 9 h. Equal volumes of PBS were added into the wells that were not treated with $\mathrm{H}_{2} \mathrm{O}_{2}$ for the same aforementioned durations. Following incubation for the specified durations, the media were removed and cells were washed three times with ice-cold PBS. The slides of cells were fixed in fresh $4 \%$ formaldehyde for $20 \mathrm{~min}$ at room temperature. Subsequently, the cells were washed three times with PBS and incubated in PBS containing 1\% (v/v) Triton X-100 and 1\% bovine serum albumin (Beyotime Institute of Biotechnology) for $1 \mathrm{~h}$ on ice to permeabilize the cells and block non-specific protein-protein interactions. The glass coverslips were removed from the 6-well plates and subsequently incubated with $30 \mu 1$ NRF2 primary antibody (cat. no. ab31163; Abcam, Cambridge, UK) diluted at 1:100 and placed in a humidified chamber overnight at $4^{\circ} \mathrm{C}$. The secondary antibody used was cyanine 3-conjugated goat anti-rabbit $\operatorname{IgG}(\mathrm{H}+\mathrm{L}$; cat. no. A01516; Beyotime Institute of Biotechnology) used at a 1:1,000 dilution for $1 \mathrm{~h}$. DAPI was used to stain the cell nuclei for $5 \mathrm{~min}$ at room temperature (cat. no. C1002; Beyotime Institute of Biotechnology). Finally, the coverslips were sealed with antifade mounting medium (Beyotime Institute of Biotechnology) and observed under fluorescence microscopy (magnification, $\mathrm{x} 40$ ). The immunofluorescence detection of NRF2 without $\mathrm{H}_{2} \mathrm{O}_{2}$ and the staining of KEAP1 were performed as described above but using a KEAP1-specific antibody (cat. no. ab139729; Abcam) also diluted at 1:100 and the same secondary antibody (cyanine 3-conjugated goat anti-rabbit IgG; H+L; cat. no. A01516).

$R T$ - $q P C R$. Cells were seeded in 6-well plates and treated with $0,0.5$ and $1.0 \mathrm{mmol} / 1 \mathrm{H}_{2} \mathrm{O}_{2}$ Total RNA was extracted from cells using TRIzol reagent (Invitrogen; Thermo Fisher Scientific, Inc.). RNA quality and quantity was measured using a NanoDrop 2000 spectrophotometer (NanoDrop Technologies; Thermo Fisher Scientific, Inc.). A total of $1 \mu \mathrm{g}$ RNA was reverse transcribed into cDNA using the PrimeScript RT reagent kit (Perfect Real Time; Takara Bio, Inc.) in a final $20 \mu$ l volume reaction, according to the manufacturer's protocol. A volume of $1 \mu \mathrm{l}$ RT reaction mixture and $9 \mu \mathrm{l}$ qPCR mixture were mixed and SYBR Green I-based RT-qPCR analyses of human KEAP1, NRF2, NQO1 and HO1 were performed by using the SYBR Premix Ex Taq system (Tli RNaseH Plus; Takara Bio, Inc.), according to the manufacturer's protocol in triplicate on an ABI 7500 system (Applied Biosystems; Thermo Fisher Scientific, Inc.). Negative RT controls were included in each assay. All primers were designed and synthesized by Sangon Biotech Co., Ltd. (Shanghai, China), as presented in Table I. GAPDH was used as a reference gene. Relative quantitative levels of samples were determined by the $2^{-\Delta \Delta C q}$ method (17).

Western blot analysis. Total protein was acquired by lysing cells in a radioimmunoprecicpitation assay buffer containing phenylmethylsulfonyl fluoride with a final concentration of $1 \mathrm{mmol} / 1$ (Beijing Cowin Biotech Co., Ltd., Beijing, China). The cytosolic and nuclear fractions of NRF2 were prepared using a NE-PER Nuclear and Cytoplasmic Extraction Reagents kit (Pierce; Thermo Fisher Scientific, Inc.). Protein extracts were obtained via centrifugation at $4^{\circ} \mathrm{C}$ and $10,000 \times \mathrm{g}$ for $10 \mathrm{~min}$; proteins were quantified using a Bicinchoninic Acid Protein assay kit (Beyotime Institute of Biotechnology). A total of $40 \mu \mathrm{g}$ total protein was electrophoretically separated via $10 \%$ SDS-PAGE and transferred to polyvinylidene fluoride membranes. To block the membranes, $5 \%$ non-fat milk in Tris-buffered saline containing $0.1 \%$ Tween-20 (TBST; $\mathrm{pH}$ 7.4-7.5) was applied at room temperature for $1 \mathrm{~h}$. Membranes were subsequently incubated overnight with anti-NRF2 (cat. no. ab31163; Abcam), anti-KEAP1 (cat. no. ab139729; Abcam), anti-NQO1 (cat. no. A180; Cell Signaling Technology, Inc., Danvers, MA, USA), anti-HO1 (cat. no. ab68477; Abcam), and anti- $\beta$-actin (cat. no. GB13001-3; Servicebio, Inc.) or lamin B primary antibodies (GB11408; Servicebio, Inc.) in blocking buffer at $4^{\circ} \mathrm{C}$ at 1:1,000 dilution. Subsequently, membranes were washed three times in TBST followed by incubation for $1 \mathrm{~h}$ with HRP-labeled goat anti-rabbit/mouse IgG (H+L) (GB23303/GB23301; Servicebio, Inc.) at a 1:2,500 dilution and washed in TBST again. Bands were visualized 


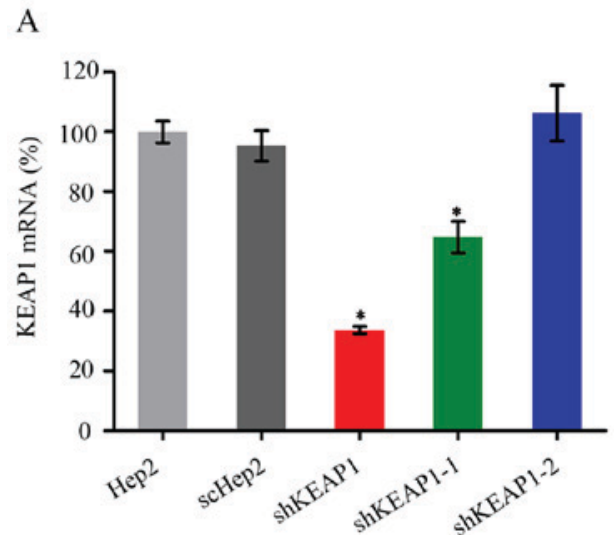

$\mathrm{C}$

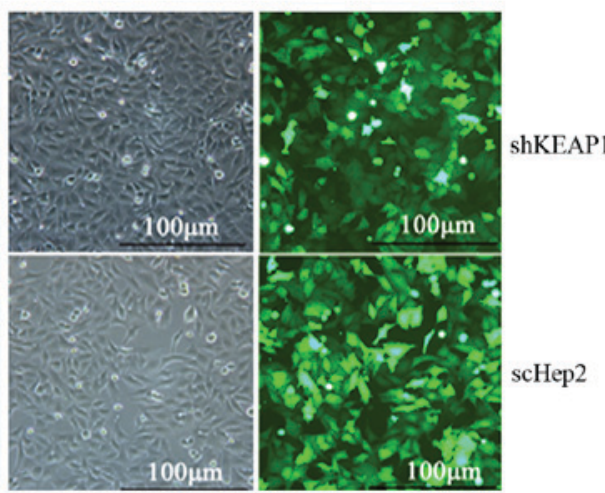

B

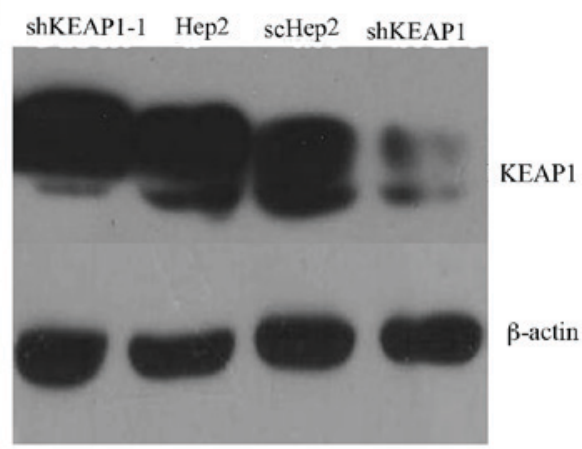

$\mathrm{D}$

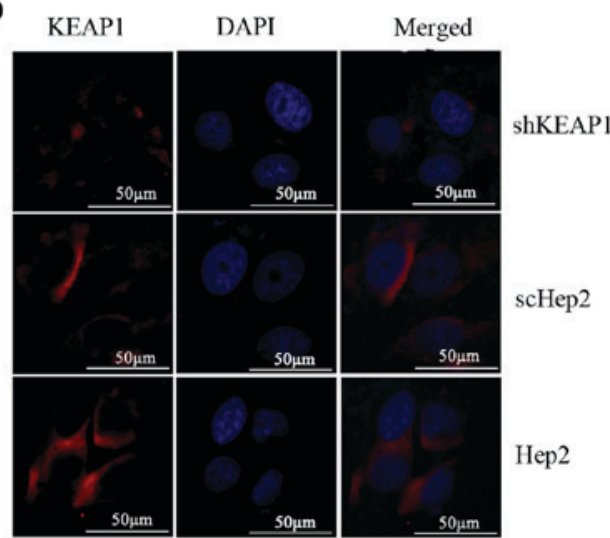

Figure 1. Confirmation of efficient KEAP1 knockdown in Hep2 cells. (A) mRNA expression levels in the three KEAP1 shRNA-transfected Hep2 cell groups. The highest reduction in KEAP1 mRNA levels compared with the scHep2 group was observed in the shKEAP1 group, with a decrease of $67 \pm 1 \%$. (B) Western blot analysis demonstrated that the protein expression of KEAP1 was markedly reduced in the shKEAP1 group compared with parent Hep2 and scHep2 groups. (C) Green fluorescent protein fluorescence was observed to determine the transfection efficiency in shKEAP1 and scHep2 cells (magnification, x40). (D) Immunofluorescence staining for KEAP1 demonstrated that KEAP1 protein expression was reduced in shKEAP1 cells compared with scHep2 and parental Hep2 cells. "P<0.05 vs. scHep2 group. KEAP1, kelch-like ECH-associated protein 1; shRNA, short hairpin RNA; scHep2, scrambled control-transfected Hep2 cells.

using hypersensitive enhanced chemiluminescence kit (cat. no. G2020; Servicebio, Inc. Wuhan China). $\beta$-actin was used for cytoplasmic extracts and lamin B used for nuclear extracts.

Statistical analysis. The significance of intergroup differences among the multiple groups was determined using one-way analysis of variance. The Least Significant Difference test was used for the post hoc test. $\mathrm{P}<0.05$ was considered to indicate a statistically significant difference. The comparisons of the cell viability among the groups were performed by Bonferroni following one-way analysis of variance. All statistical analyses were conducted using the SPSS 17.0 statistical package (SPSS, Inc., Chicago, IL, USA). The experiments were repeated at least three times and data are presented as the mean \pm standard deviation.

\section{Results}

Confirmation of efficient KEAPI knockdown. In order to establish a stable shKEAP1 Hep2 cell line with effective knockdown of the KEAP1 gene, Hep2 cells were transfected with three KEAP1 shRNAs and one scrambled shRNA expression lentiviral plasmids bearing GFP, which was used to assess the efficacy of transfection. RT-qPCR was performed on exponentially growing cells to determine the expression levels of KEAP1 following $72 \mathrm{~h}$ transduction. Of the three
shKEAP1 sequences, one exhibited the highest knockdown efficiency, which reduced KEAP1 mRNA expression levels by up to $67 \pm 1 \%$ compared with the Hep2 cells transfected with the other shRNAs $(\mathrm{P}<0.05$; Fig. 1A). Based on this, this sequence was selected to establish a stable shKEAP1 Hep2 cell line with puromycin for 2 weeks. Subsequently, the protein expression levels of KEAP1 in shKEAP1 Hep2 and scHep2 cell lines were measured by western blotting. KEAP1 protein expression levels were markedly reduced following KEAP1 knockdown using the shKEAP1 construct (Fig. 1B). Furthermore, the transfection efficiency of scHep2 cells was demonstrated to be equivalent to that of shHep2, which was $\sim 80 \%$ (Fig. 1C). Immunofluorescence staining demonstrated that KEAP1 protein was primarily expressed in the cytoplasm. Weak fluorescence and bands were detected within the shKeap1 group, which were markedly lower compared with in scHep2 and Hep2 cells; however, within the control groups, expression levels were similar (Fig. 1D), indicating that a stable and effective shKEAP1 Hep2 cell line was established.

KEAP1 knockdown increases the expression of NRF2, NQO1 and HOI in Hep 2 cells. The effects of KEAP1 knockdown on the mRNA and protein expression of NRF2 and NRF2-associated genes were investigated. The results demonstrated that shRNA-mediated depletion of KEAP1 was associated with significant increases in the mRNA expression 
A

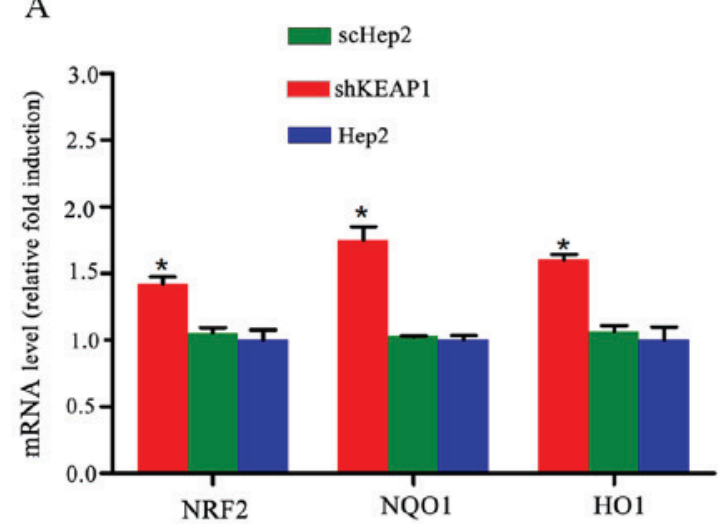

$\mathrm{C}$

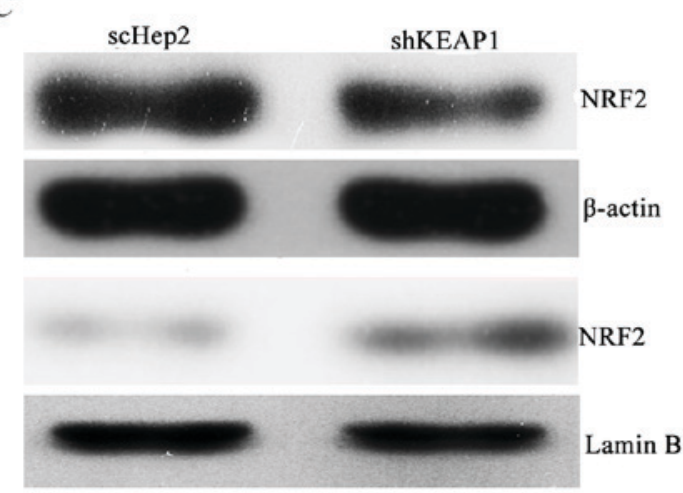

B

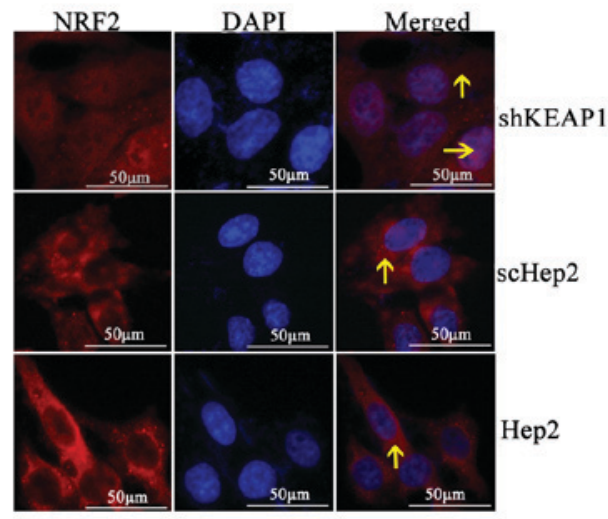

$\mathrm{D}$

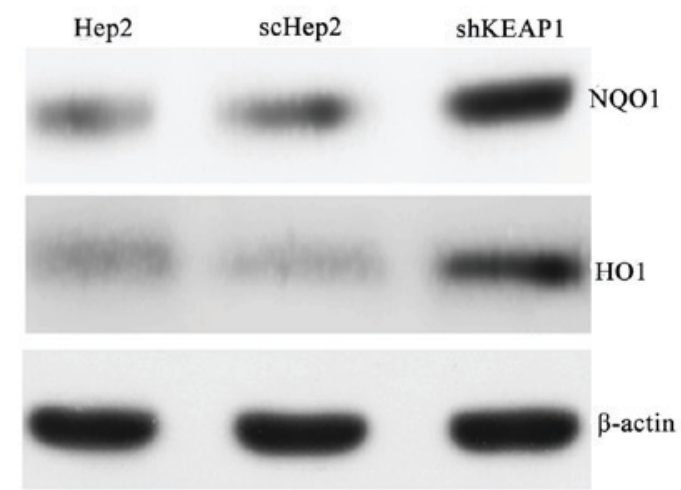

Figure 2. Effect of KEAP1 knockdown on the expression of NRF2 and downstream targets. (A) mRNA expression levels of NRF2, NQO1 and HO1 in Hep2, scHep2 and shKEAP1 Hep2 cells. Expression levels of NRF2, NQO1 and HO1 were increased following the knockdown of KEAP1 in Hep2 cells. (B) Representative NRF2 immunofluorescence staining images indicate that NRF2 translocated into the nuclei from the cytoplasm following knockdown of KEAP1 in Hep2 cells (magnification, x40). (C) Western blotting demonstrated that nuclear NRF2 protein expression levels were elevated, while cytoplasmic NRF2 protein expression levels were reduced, following the knockdown of KEAP1 in Hep2 cells. (D) Western blotting demonstrated that total NQO1 and HO1 protein expression levels were increased within shKEAP1 Hep2 cells, compared with the scHep2 group. *P<0.05 vs. scHep2 group. The arrows indicate NRF2. KEAP1, kelch-like ECH-associated protein 1; NRF2, nuclear factor erythroid 2-related factor 2; NQO1, NAD(P)H quinone oxidoreductase 1; HO1, heme oxygenase 1; scHep2, scrambled control-transfected Hep2 cells; sh, short hairpin RNA.

levels of NRF2, NQO1 and HO1 by $1.42 \pm 0.05-, 1.75 \pm 0.10-$ and $1.59 \pm 0.07$-fold, respectively, compared with the scHep2 group (Fig. 2A). Notably, immunofluorescence staining demonstrated that the expression levels of nuclear NRF2 were low, with NRF2 primarily located in the cytoplasm within parent Hep2 cells; however, NRF2-reactive fluorescent signals were observed within the nucleus of shKEAP1 cells, indicating that KEAP1 knockdown may cause NRF2 migration into the nuclei (Fig. 2B). Furthermore, western blot analysis demonstrated that delivery of specific shKEAP1 by lentiviral transduction reduced the cytosolic levels of NRF2, which was observed alongside a marked increase in nuclear NRF2 levels (Fig. 2C). Total protein levels of NQO1 and HO1 were markedly elevated in shKEAP1 Hep2 cells compared with the scHep2 cell line and the blank control Hep2 group (Fig. 2D).

Effect of KEAP1 knockdown on oxidative damage in Hep2 cells. To investigate the role of KEAP1 knockdown against oxidative damage in the Hep2 cell line, the expression of this system and the resistance of the cells to oxidative stresses induced by $\mathrm{H}_{2} \mathrm{O}_{2}$ were analyzed. Immunofluorescence analysis demonstrated that when cultured shKEAP1 Hep2 cells were treated with 0.5 and $1 \mathrm{mmol} / 1 \mathrm{H}_{2} \mathrm{O}_{2}$ for $6 \mathrm{~h}$, NRF2 markedly migrated into the cell nucleus compared with in scHep2 cells.
In addition, the expression of NRF2 appeared to be markedly higher in response to the concentration of $1 \mathrm{mM} / 11 \mathrm{H}_{2} \mathrm{O}_{2}$ for $6 \mathrm{~h}$ compared with $0.5 \mathrm{mmol} / 1$ (Fig. 3A-C). As presented in Figs. 3D and $\mathrm{E}$, and 4 , the results of RT-qPCR analysis and western blotting demonstrated consistencies with immunofluorescence analysis; the increases in the NRF2, NQO1 and HO1 transcript and protein levels demonstrated time- and dose-dependency within the knockdown cell line. Knockdown of KEAP1 via shRNA in Hep2 cells increased the NRF2, NQO1 and HO1 mRNA expression levels by 1.99, 2.32 and 1.91-fold respectively, compared with scHep2 cells when treated with $0.5 \mathrm{mM} / 1$ $\mathrm{H}_{2} \mathrm{O}_{2}$ for $6 \mathrm{~h}$ (Figs. 3D, 4A and B). At the concentration of $1 \mathrm{mM} / 1 \mathrm{H}_{2} \mathrm{O}_{2}$ for $9 \mathrm{~h}$, NRF2, NQO1 and $\mathrm{HO} 1 \mathrm{mRNA}$ levels were 2.85, 3.47 and 2.96-fold higher in the KEAP1 knockdown group, respectively, compared with in the scHep2 cells. mRNA expression levels were analyzed upon exposure to $0.5 \mathrm{mmol} / \mathrm{l}$ $\mathrm{H}_{2} \mathrm{O}_{2}$ for 6 and $9 \mathrm{~h}$. NRF2, NQO1 and HO1 were all expressed highly within shKEAP1 cells compared with in scHep2 cells at both concentrations. Western blot analyses revealed that the protein expression levels of nuclear NRF2, and total NQO1 and HO1, were markedly upregulated in shKEAP1 Hep2 cells compared with in scHep2 control cells at the concentration of 0.5 and $1 \mathrm{mmol} / 1 \mathrm{H}_{2} \mathrm{O}_{2}$ for $6 \mathrm{~h}$ (Figs. 3E and 4C). Supporting the results of immunofluorescence, KEAP1 knockdown led to 
A

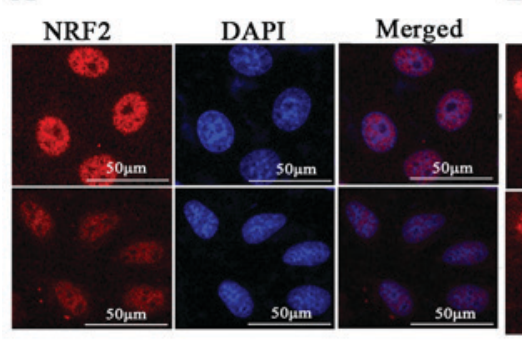

$\mathrm{H}_{2} \mathrm{O}_{2}(0.5 \mathrm{mmol} / \mathrm{l})+6 \mathrm{~h}$
B

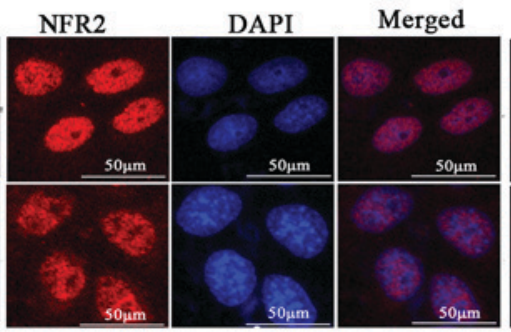

$\mathrm{H}_{2} \mathrm{O}_{2}(1.0 \mathrm{mmol} / \mathrm{l})+6 \mathrm{~h}$
C

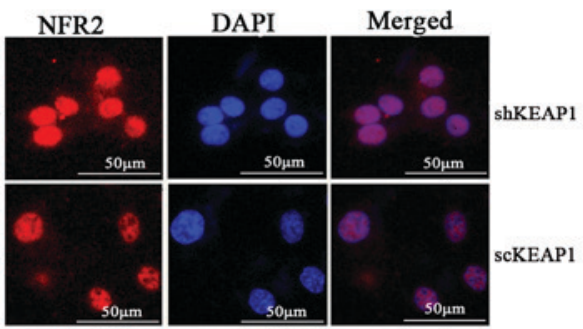

$\mathrm{H}_{2} \mathrm{O}_{2}(0.5 \mathrm{mmol} / \mathrm{l})+9 \mathrm{~h}$
D

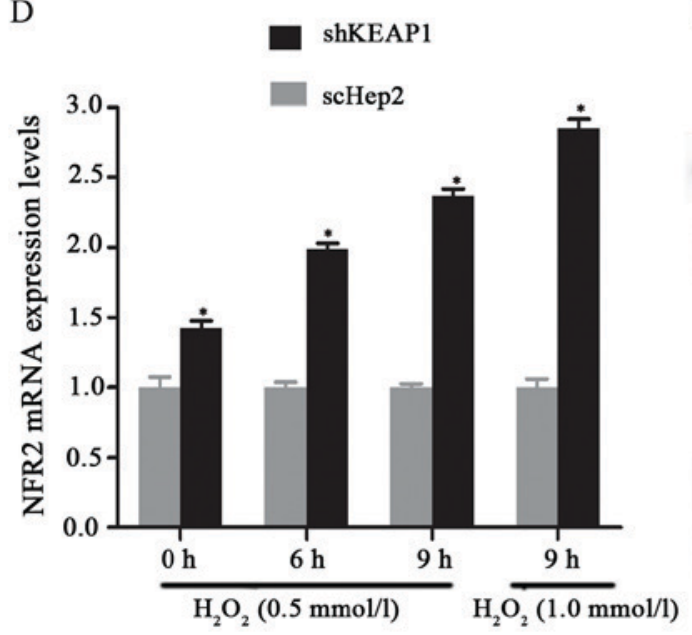

E

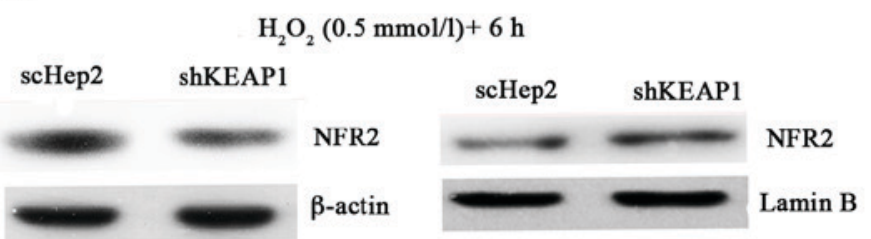

$\mathrm{H}_{2} \mathrm{O}_{2}(1.0 \mathrm{mmol} / \mathrm{l})+6 \mathrm{~h}$

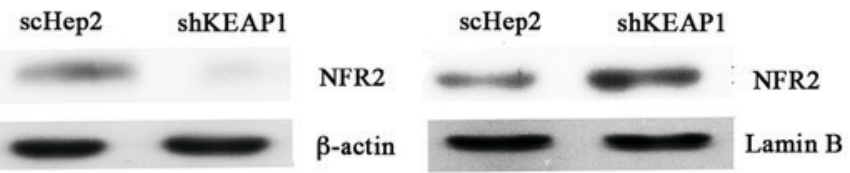

Figure 3. NRF2 translocated into the nuclei and aggregated as granules in shKEAP1 and scHep2 cells following exposure to $\mathrm{H}_{2} \mathrm{O}_{2}$ in a dose-dependent manner when cultured for 6 and $9 \mathrm{~h}$. Representative images of NRF2 immunostaining in shKEAP1 and scHep2 cells following treatment with (A) 0.5 mmol/1 $\mathrm{H}_{2} \mathrm{O}_{2}$ for $6 \mathrm{~h}$, (B) $1 \mathrm{mmol} / 1 \mathrm{H}_{2} \mathrm{O}_{2}$ for $6 \mathrm{~h}$ and (C) $0.5 \mathrm{mmol} / 1 \mathrm{H}_{2} \mathrm{O}_{2}$ for $9 \mathrm{~h}$ (magnification, x40). (D) NRF2 mRNA levels in shKEAP1 and scHep2 cells increased in a dose- and time-dependent manner following exposure to $\mathrm{H}_{2} \mathrm{O}_{2} .{ }^{*} \mathrm{P}<0.05$ vs. scHep2 group. (E) Western blot analysis indicated that NRF2 protein expression levels in the cytoplasm were decreased with increasing concentrations of $\mathrm{H}_{2} \mathrm{O}_{2}$ in shKEAP1 and scHep 2 cells, but were higher within scHep2 cells than in shKEAP1 cells. The opposite was observed in the nucleus. NRF2, nuclear factor erythroid 2-related factor 2; sh, short hairpin RNA; KEAP1, kelch-like ECH-associated protein 1; scHep2, scrambled control-transfected Hep2 cells; $\mathrm{H}_{2} \mathrm{O}_{2}$, hydrogen peroxide.

a reduction in the protein expression levels of NRF2 within the cytoplasm and an increase within the nuclei at 0.5 and $1 \mathrm{mM} / 1$ $\mathrm{H}_{2} \mathrm{O}_{2}$ concentrations, compared with scHep2 cells (Fig. 3E). The expression patterns of NQO1 and $\mathrm{HO} 1$ were similar to the nuclear NRF2 in shKEAP1 Hep2 cells upon exposure to $\mathrm{H}_{2} \mathrm{O}_{2}$ (Fig. 4C).

Effects of KEAP1 knockdown on Hep2 cell function. As the expression levels of NRF2, NQO1 and HO1 were altered within NRF2-activated Hep2 cells, the effects of KEAP1 knockdown on cell function were subsequently investigated. Cell viability decreased in a dose-dependent manner within the shKEAP1 and scHep2 cell lines with increasing doses of $\mathrm{H}_{2} \mathrm{O}_{2}$ with the range of final concentrations from $0-1 \mathrm{mM} / \mathrm{l}$ for $24 \mathrm{~h}$. At concentrations of $0.25,0.5$ and $0.75 \mathrm{mM} / 1$, the mean relative viabilities of shKEAP1 and scHep2 cells were 75 and 51,33 and 12 , and 18 and $6 \%$, respectively. It was observed that transfected shKEAP1 Hep2 cells demonstrated significantly higher cell viabilities following $\mathrm{H}_{2} \mathrm{O}_{2}$ treatment compared with in the scHep2 group, as demonstrated in Fig. 5. To validate the results obtained from the CCK- 8 assay and to investigate whether apoptosis served a role against oxidative stress injury, the percentage of the apoptotic cells was analyzed by flow cytometry. Cells were cultured with varying concentrations of $\mathrm{H}_{2} \mathrm{O}_{2}(0,0.1$ and $0.25 \mathrm{mmol} / \mathrm{l})$ for $24 \mathrm{~h}$. Marked increases were observed in the apoptosis rates of scHep2 cells at 0.1 and $0.25 \mathrm{mmol} / \mathrm{l} \mathrm{H}_{2} \mathrm{O}_{2}$ from 31.8 and $45.3 \%$, while 14.1 and $27.9 \%$ in shKEAP1 cells (Fig. 6).

\section{Discussion}

The association between oxidative damage and cancer has been increasingly emphasized. High antioxidant levels have been reported within the serum and tissue samples of numerous patients with cancer. Suzuki et al reported that increasing the intake of antioxidants, such as renieratene, vitamin $\mathrm{C}$ and vitamin $\mathrm{E}$, may not only reduce the risk of developing head and neck cancer of the general population, but also those who smoke and consume alcohol (18). One study demonstrated that cells exhibit elevated sensitivity to the external harmful materials, when the activities of antioxidants and enzymes are inhibited (19). These findings have demonstrated that oxidative damage may exert notable effects in the process of the cancer occurrence, progression and treatment.

When confronted with oxidative stress, antioxidative systems within the body are activated to remove oxidative stress-inducing substances. Knockout of the NRF2 gene in mice revealed a reduction of antioxidant enzymes, including glutathione S-transferase and HO1, and an increased sensitivity of mice to carcinogens as knockout mice exhibited higher 
A

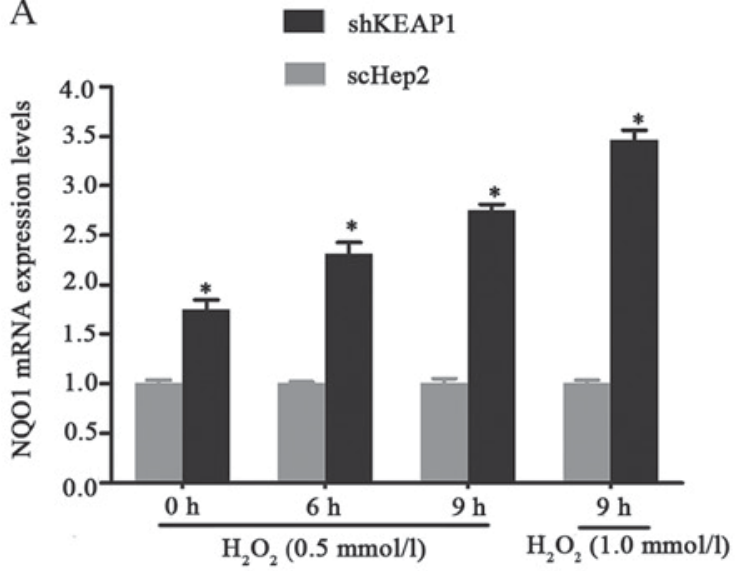

B

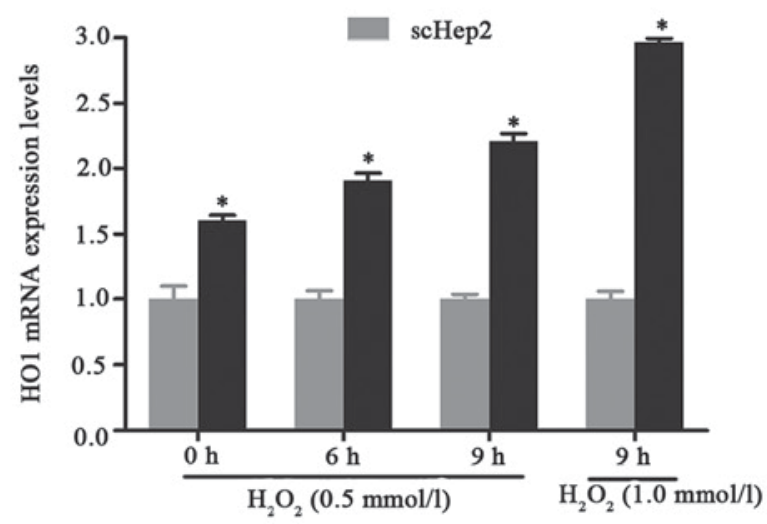

C
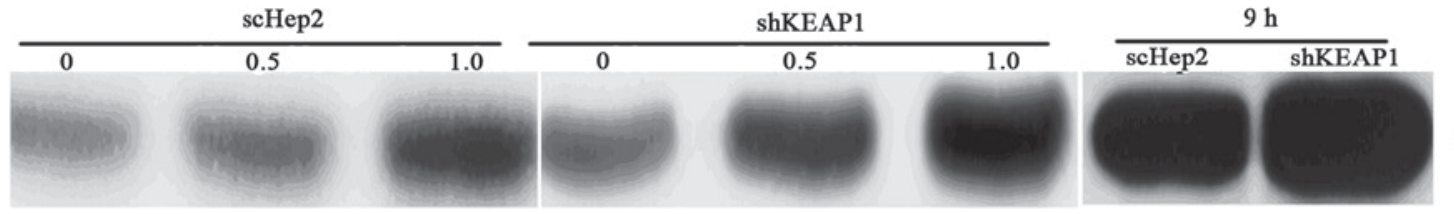

NQO1

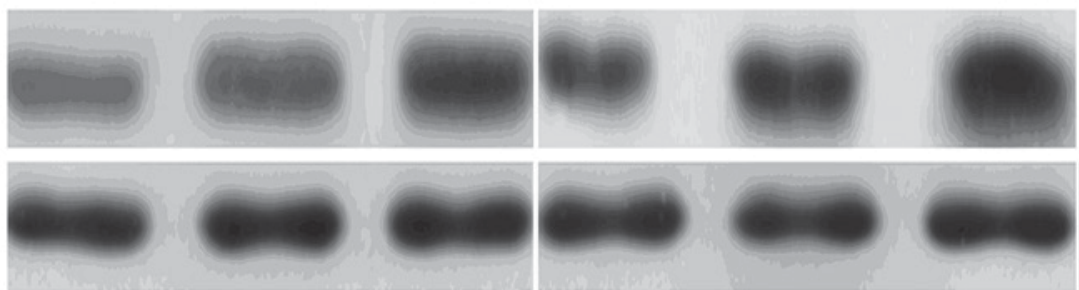

$\mathrm{H}_{2} \mathrm{O}_{2}(0.5 \mathrm{mmol} / \mathrm{l})+6 \mathrm{~h}$

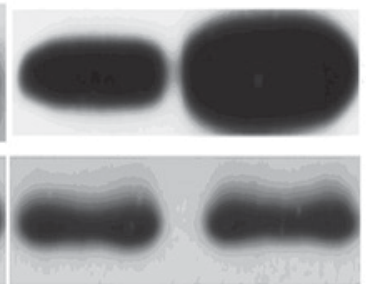

HO1

$\beta$-actin

$\mathrm{H}_{2} \mathrm{O}_{2}(1.0 \mathrm{mmol} / \mathrm{l})$

Figure 4. Effect of KEAP1 knockdown on $\mathrm{H}_{2} \mathrm{O}_{2}$-induced alterations in the expression of NQO1 and HO1. mRNA levels of (A) NQO1 and (B) HO1 within shKEAP1 Hep2 cells were increased in a dose- and time-dependent manner following exposure to $\mathrm{H}_{2} \mathrm{O}_{2}$. KEAP1 knockdown upregulated the expression levels of NQO1 and HO1 compared with in scHep2 cells. "P<0.05 vs. scHep2 group. (C) Western blotting indicated that the protein expression levels of NQO1 and $\mathrm{HO} 1$ within the shKEAP1 Hep2 cells were increased with increasing concentrations of $\mathrm{H}_{2} \mathrm{O}_{2}$ and with increasing incubation durations. There were also marked differences between shKEAP1 and scHep2 cells. KEAP1, kelch-like ECH-associated protein $1 ; \mathrm{H}_{2} \mathrm{O}_{2}$, hydrogen peroxide; $\mathrm{NQO1}$, NAD(P)H quinone oxidoreductase 1; HO1, heme oxygenase 1; sh, short hairpin RNA; scHep2, scrambled control-transfected Hep2 cells.

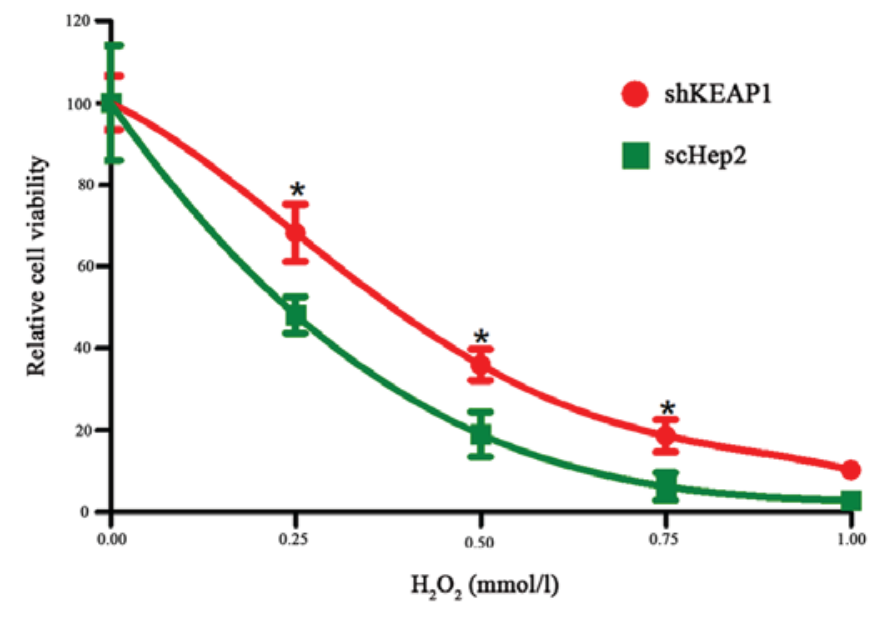

Figure 5. shKEAP1 cells exhibited a significantly higher cell viability following $\mathrm{H}_{2} \mathrm{O}_{2}$ exposure compared with scHep2 cells. "P<0.05 vs. scHep2 group at same concentration of $\mathrm{H}_{2} \mathrm{O}_{2}$. sh, short hairpin RNA; KEAP1, kelch-like ECH-associated protein $1 ; \mathrm{H}_{2} \mathrm{O}_{2}$, hydrogen peroxide; scHep2, scrambled control-transfected Hep2 cells.

incidences of cancer compared with wild-type mice (20). Research has also indicated that interfering with KEAP1 gene expression may upregulate the expression of NRF2; Singh et al reported that within lung cancer cells with low expression levels of KEAP1, the nuclear NRF2 expression levels were significantly higher compared with lung cancer cells with high KEAP1 expression. NQO1 expression was increased in lung cancer cells with lower KEAP1 expression, compared with cells with high KEAP1 expression. In addition, NRF2 and its downstream genes were demonstrated to be downregulated via transfection of NRF2-siRNA into cells or through increasing KEAP1 expression (10). These findings have also been observed within pancreatic cancer cell lines, where KEAP1 was also inversely associated with NRF2 and its downstream genes, including NQO1 and HO1. Therefore, high expression levels of KEAP1 may lead to low expression levels of NRF2 and antioxidants. Elevated levels of KEAP1 expression may cause the expansion ability of cells to decrease (21). In addition, these findings were also verified within ovarian carcinoma cells. Following the introduction of KEAP1-siRNA into ovarian carcinoma cells, the expression levels of KEAP1 mRNA decreased by $60 \%$ and the target protein NQO1 was markedly lowered, which accelerated the cell growth (11). Jung and Kwak identified the same phenomenon within colorectal cells. Specific KEAP1-shRNA was transfected into colorectal cells to reduce the expression levels of KEAP1 mRNA by 50\%, 
A

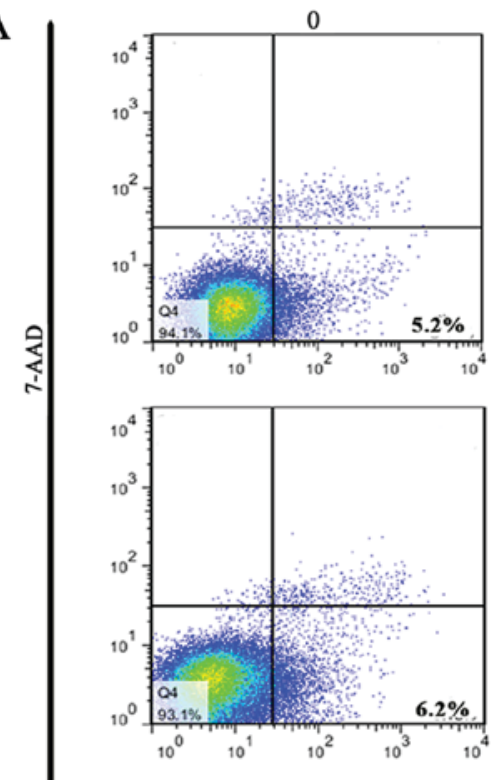

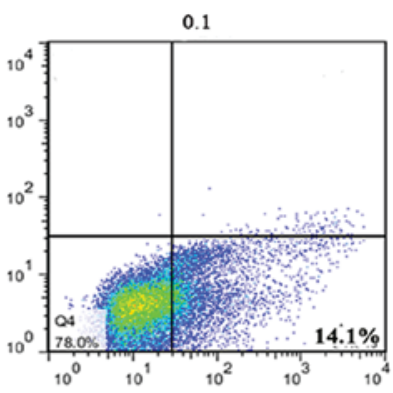

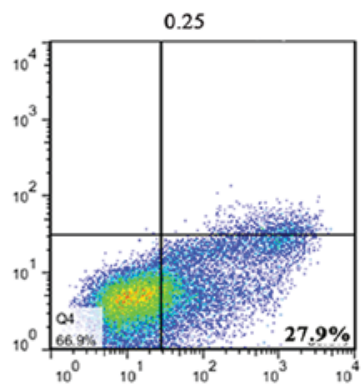

$\mathrm{H}_{2} \mathrm{O}_{2}(\mathrm{mmol} / \mathrm{l})$
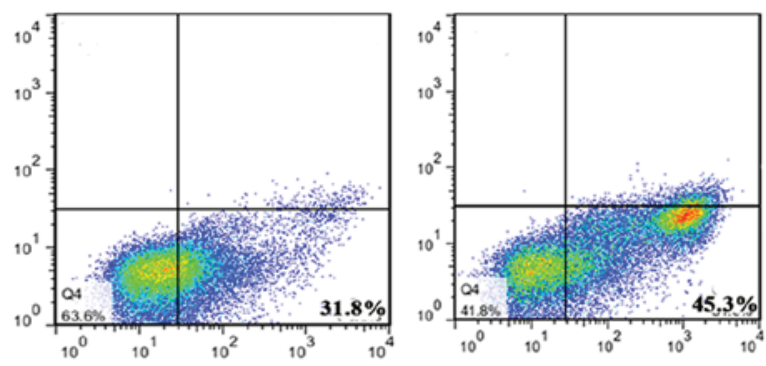

ShKEAP1

scHep2

Annexin V-APC

B

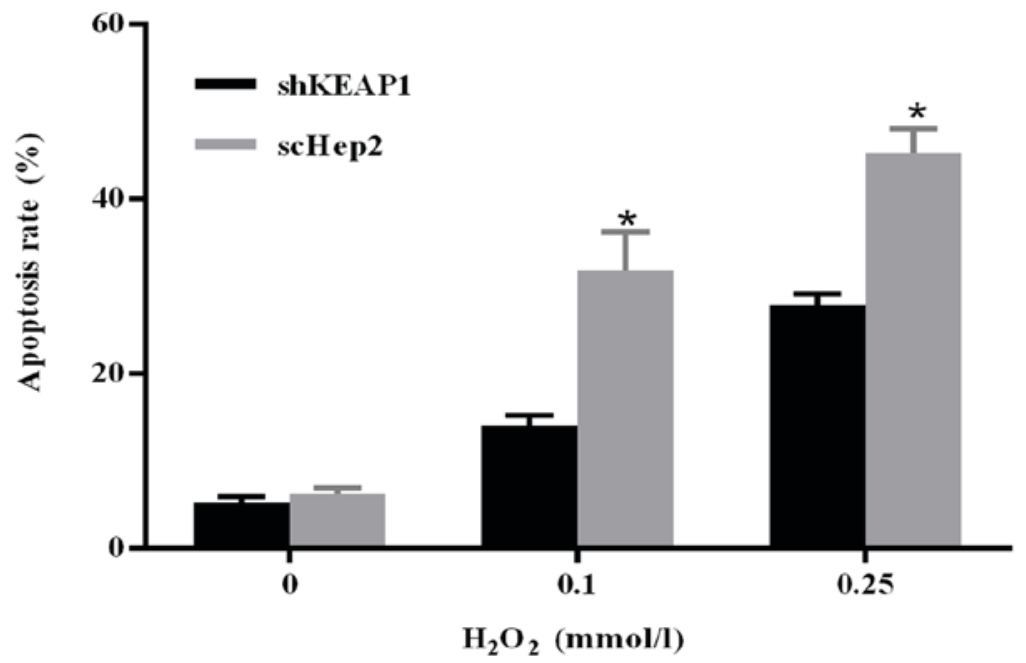

Figure 6. Effect of KEAP1 knockdown on $\mathrm{H}_{2} \mathrm{O}_{2}$-induced alterations in $\mathrm{Hep} 2$ cell apoptosis. (A) $\mathrm{H}_{2} \mathrm{O}_{2}$ evidently induced cell apoptosis from 14.1 to 27.9 and 31.8 to $45.3 \%$ in shKEAP1 and scHep2 cells, respectively. (B) The apoptotic rate in shKEAP1 Hep2 cells was lower compared with in scHep2 cells. ${ }^{*} \mathrm{P}<0.05$ vs. the shKEAP1 at the corresponding $\mathrm{H}_{2} \mathrm{O}_{2}$ concentration. KEAP1, kelch-like ECH-associated protein $1 ; \mathrm{H}_{2} \mathrm{O}_{2}$, hydrogen peroxide; $\mathrm{H}_{2} \mathrm{O}_{2}$, hydrogen peroxide; sh, short hairpin RNA; scHep2, scrambled control-transfected Hep2 cells; APC, allophycocyanin; 7-AAD, 7-aminoactinomycin D. The lower right-hand side quadrant was considered to indicate apoptotic cells.

resulting in the marked upregulation of nuclear translocation of NRF2 and the expression of its target proteins, including NQO1 and HO1 (22).

In the present study, the experimental results regarding the role of the KEAP1-NRF2-ARE signaling pathway within Hep2 cells were consistent with the above motioned studies. A highly effective shKEAP1 stable Hep2 cell line was established in the present study. Low expression levels of NQO1 and HO1 were detected within normal Hep2 cells, while shKEAP1 cells exhibited increased expression levels. Importantly, the findings of the present study confirmed that the downregulation of KEAP1 was associated with the migration of NRF2 from the cytoplasm to the nuclei. This demonstrated that the activation of NRF2 was associated with high expression levels of NQO1 and HO1. In addition, the effects of KEAP1 knockdown on the proliferation of Hep2 cells were analyzed. The results demonstrated that the cells of the shKEAP1 group exhibited a marked increase in their proliferation compared with the scHep2 control group. Notably, the cell viability of shKEAP1 Hep2 cells was greater compared with in scHep2 cells from 48 to $96 \mathrm{~h}$ following the transfection of KEAP1-shRNA (data not shown). This indicated that the effects of KEAP1 knockdown via lentiviral transfection may be long lasting. To investigate the mechanisms involved in this process, the apoptotic rate was measured within the scHep2 and shKEAP1 Hep2 cell lines. Marked alterations $24 \mathrm{~h}$ post-transfection between the shKEAP1 and control groups were not observed at $0 \mathrm{mmol} / 1 \mathrm{H}_{2} \mathrm{O}_{2}$; however, at 0.1 and $0.25 \mathrm{mmol} / 1 \mathrm{H}_{2} \mathrm{O}_{2}$ the apoptosis rate was higher within scHep2 cells compared with in shKEAP1 Hep2 cells. These results demonstrated that the inhibition of KEAP1 expression within Hep2 cells may reduce cell apoptosis to improve survivability. In addition, similar findings have been observed in other cell types. Li et al reported that NRF2 was primarily located within nuclei 
and that overexpression of NRF2 may promote proliferation in endometrial cancer cells (23). Ma et al revealed that the downregulation of NRF2 reduced the expression levels of NQO1 and HO1 within cervical cancer cells. Furthermore, the volume of shNRF2-transfected cervical cancer cell xenograft tumors was lower compared with wild-type NRF2 expression cell xenograft tumors (24).

Numerous studies have demonstrated that there are close associations between $\mathrm{H}_{2} \mathrm{O}_{2}$ and the KEAP1-NRF2-ARE signaling pathway. Following the addition of $\mathrm{H}_{2} \mathrm{O}_{2}$ to astrocyte cells, the KEAP1/NRF2 system was activated and NRF2 migrated into the nuclei from the cytoplasm. The expression levels of NRF2 and its target protein, NQO1, were notably upregulated, which was positively associated with increasing $\mathrm{H}_{2} \mathrm{O}_{2}$ concentrations and the duration of treatment $(25,26)$. Pi et al (27) also demonstrated that $\mathrm{H}_{2} \mathrm{O}_{2}$ markedly induced nuclear NRF2 expression and reduced cytoplasmic NRF2 expression. The longer the duration of treatment and the higher the concentration was, the higher expression levels of NQO1 and nuclear NRF2 (27). Additionally, an in vivo study revealed that nuclear NRF2 accumulated within living tissues induced by $\mathrm{H}_{2} \mathrm{O}_{2}$ in a dose-dependent manner. Collectively, these previous studies revealed that $\mathrm{H}_{2} \mathrm{O}_{2}$ may activate the KEAP1-NRF2-ARE signaling pathway and affect NRF2 nuclear transfer in a time- and dose-dependent manner. In the present study, research into NRF2 nuclear migration was performed using varying $\mathrm{H}_{2} \mathrm{O}_{2}$ concentrations and durations of treatment. As expected, marked nuclear transfer of NRF2 was observed in response to $\mathrm{H}_{2} \mathrm{O}_{2}$, and this phenomenon was more profound within shKEAP1 Hep2 cells compared with scHep2 cells. The upregulation of nuclear NRF2, NQO1 and $\mathrm{HO} 1$ may have reinforced the antioxidant capacity in Hep2 cells. Habib et al reported that following the addition of varying concentrations of $\mathrm{H}_{2} \mathrm{O}_{2}$ to a breast carcinoma cell line, NQO1 and HO1 expression, and nuclear NRF2 expression, were notably increased in a time- and dose-dependent manner (28).

The present study identified the typical KEAP1-NRF2-ARE signaling pathway within Hep2 cells. Suppression of KEAP1 may lead to a decline in cytoplasmic NRF2 and migration of NRF2 to the nuclei, which is a core factor promoting the expression of antioxidants, including NQO1 and HO1. It may be advantageous to inhibit the antioxidant activity of the Hep2 cells via the inactivation of the KEAP1-NRF2-ARE signal transduction pathway (29). Once the signaling pathway was inactivated, the cytoprotective proteins, including NQO1 and $\mathrm{HO} 1$ are downregulated and survival of cells decreased. This may be useful for inhibiting the growth of cancer. The results of the present study may contribute to the prevention, diagnosis and treatment of cancer.

\section{Acknowledgements}

The present study was supported by Science and Technology Commission of Shanghai Municipality (grant no. 15401971600).

\section{Funding}

No funding was received.

\section{Availability of data and materials}

All data generated or analyzed during this study are included in this published article.

\section{Authors' contributions}

LC, CL, HW and PH conceived and designed the study. CL, YZ, JC, YY and MC performed the experiments, and completed the acquisition, analysis and interpretation of data for the work. LC and CL wrote the paper. HW and PH reviewed and edited the manuscript. All authors read and approved the manuscript.

\section{Ethics approval and consent to participate}

Not applicable.

\section{Consent for publication}

Not applicable.

\section{Competing interests}

The authors declare that they have no competing interests.

\section{References}

1. Jałoszyński P, Jaruga P, Oliński R, Biczysko W, Szyfter W, Nagy E, Möller L and Szyfter K: Oxidative DNA base modifications and polycyclic aromatic hydrocarbon DNA adducts in squamous cell carcinoma of larynx. Free Radic Res 37: 231-240, 2003.

2. Seven A, Civelek S, Inci E, Inci F, Korkut N and Burçak G: Evaluation of oxidative stress parameters in blood of patients with laryngeal carcinoma. Clin Biochem 32: 369-373, 1999.

3. Dwivedi R, Raturi D, Kandpal N, Dwivedi R, Singh R and Puri V: Oxidative stress in patients with laryngeal carcinoma. Indian J Cancer 45: 97-99, 2008.

4. Inci E, Civelek S, Seven A, Inci F, Korkut N and Burçax G: Laryngeal cancer: In relation to oxidative stress. Tohoku J Exp Med 200: 17-23, 2003

5. Motohashi H and Yamamoto M: Nrf2-Keap1 defines a physiologically important stress response mechanism. Trends Mol Med 10: 549-557, 2004.

6. Uruno A and Motohashi H: The Keap1-Nrf2 system as an in vivo sensor for electrophiles. Nitric Oxide 25: 153-160, 2011.

7. Awadallah NS, Dehn D, Shah RJ, Russell Nash S, Chen YK, Ross D, Bentz JS and Shroyer KR: NQO1 expression in pancreatic cancer and its potential use as a biomarker. Appl Immunohistochem Mol Morphol 16: 24-31, 2008.

8. Dunn L, Midwinter RG, Ni J, Hamid HA, Parish CR and Stoker R: New insights into intracellular locations and functions of heme oxygenase-1. Antioxid Redox Signal 20: 1723-1742, 2014.

9. Kensler TW and Wakabayashi N: Nrf2: Friend or foe for chemoprevention? Carcinogenesis 31: 90-99, 2010.

10. Singh A, Misra V, Thimmulappa RK, Lee H, Ames S, Hoque MO, Herman JG, Baylin SB, Sidransky D, Gabrielson E, et al: Dysfunctional KEAP1-NRF2 interaction in non-small-cell lung cancer. PLoS Med 3: e420, 2006.

11. Konstantinopoulos PA, Spentzos D, Fountzilas E, Francoeur N, Sanisetty S, Grammatikos AP, Hecht JL and Cannistra SA: Keap1 mutations and Nrf2 pathway activation in epithelial ovarian cancer. Cancer Res 71: 5081-5089, 2011.

12. Kim JH, Choi YK, Lee KS, Cho DH, Baek YY, Lee DK, Ha KS, Choe J, Won MH, Jeoung D, et al: Functional dissection of Nrf2-dependent phase II genes in vascular inflammation and endotoxic injury using Keapl siRNA. Free Radic Biol Med 53: 629-640, 2012. 
13. Wakabayashi N, Itoh K, Wakabayashi J, Motohashi H, Noda S, Takahashi S, Imakado S, Kotsuji T, Otsuka F, Roop DR, et al: Keapl-null mutation leads to postnatal lethality due to constitutive Nrf2 activation. Nat Genet 35: 238-245, 2003.

14. Spanou C, Stagos D, Aligiannis N and Kouretas D: Influence of potent antioxidant leguminosae family plant extracts on growth and antioxidant defense system of Hep2 cancer cell line. J Med Food 13: 149-55, 2010.

15. Spanou C, Stagos D, Aligiannis N and Kouretas D: Influence of potent antioxidant leguminosae family plant extracts on growth and antioxidant defense system of Hep2 cancer cell line. J Med Food 13: 149-155, 2010.

16. Jewett A, Wang MY, Teruel A, Poupak Z, Bostanian Z and Park NH: Cytokine dependent inverse regulation of CD54 (ICAM1) and major histocompatibility complex class I antigens by nuclear factor kappaB in HEp2 tumor cel: Effect on the function of natural killer cells. Hum Immunol 64: 505-520, 2003.

17. Livak KJ and Schmittgen TD: Analysis of relative gene expression data using real-time quantitative PCR and the 2(-Delta Delta C(T)) method. Methods 25: 402-408, 2001.

18. Suzuki T, Wakai K, Matsuo K, Hirose K, Ito H, Kuriki K, Sato S, Ueda R, Hasegawa Y and Tajima K: Effect of dietary antioxidants and risk of oral, pharyngeal and laryngeal squamous cell carcinoma according to smoking and drinking habits. Cancer Sci 97: 760-767, 2006

19. Khor TO, Huang MT, Prawan A, Liu Y, Hao X, Yu S, Cheung WK, Chan JY, Reddy BS, Yang CS and Kong AN: Increased susceptibility of Nrf2 knockout mice to colitis-associated colorectal cancer. Cancer Prev Res (Phila) 1: 187-191, 2008.

20. Iida K, Itoh K, Kumagai Y, Oyasu R, Hattori K, Kawai K, Shimazui T, Akaza $\mathrm{H}$ and Yamamoto M: Nrf2 is essential for the chemopreventive efficacy of oltipraz against urinary bladder carcinogenesis. Cancer Res 64: 6424-6431, 2004.

21. Lister A, Nedjadi T, Kitteringham NR, Campbell F, Costello E, Lloyd B, Copple IM, Williams S, Owen A, Neoptolemos JP, et al: Nrf2 is overexpressed in pancreatic cancer: Implications for cell proliferation and therapy. Mol Cancer 10: 37, 2011.
22. Jung KA and Kwak MK: Enhanced 4-hydroxynonenal resistance in KEAP1 silenced human colon cancer cells. Oxid Med Cell Longev 2013: 423965, 2013.

23. Li K, Zhong C, Wang B, He J and Bi J: Nrf2 expression participates in growth and differentiation of endometrial carcinoma cells in vitro and in vivo. J Mol Histol 45: 161-167, 2014.

24. Ma X, Zhang J, Liu S, Huang Y, Chen B and Wang D: Nrf2 knockdown by shRNA inhibits tumor growth and increases efficacy of chemotherapy in cervical cancer. Cancer Chemother Pharmacol 69: 485-494, 2012.

25. Dell'Orco M, Milani P, Arrigoni L, Pansarasa O, Sardone V, Maffioli E, Polveraccio F, Bordoni M, Diamanti L, Ceroni M, et al: Hydrogen peroxide-mediated induction of SOD1 gene transcription is independent from Nrf2 in a cellular model of neurodegeneration. Biochim Biophys Acta 1859: 315-323, 2016.

26. Erlank H, Elmann A, Kohen R and Kanner J: Polyphenols activate $\mathrm{Nrf} 2$ in astrocytes via $\mathrm{H} 2 \mathrm{O} 2$, semiquinones, and quinones. Free Radic Biol Med 51: 2319-2327, 2011.

27. Pi J, Qu W, Reece JM, Kumagai Y and Waalkes MP: Transcription factor Nrf2 activation by inorganic arsenic in cultured keratinocytes: Involvement of hydrogen peroxide. Exp Cell Res 290: 234-245, 2003

28. Habib E, Linher-Melville K, Lin HX and Singh G: Expression of $\mathrm{xCT}$ and activity of system xc(-) are regulated by NRF2 in human breast cancer cells in response to oxidative stress. Redox Biol 5: 33-42, 2015.

29. Chen J, Yu Y, Ji T, Ma R, Chen M, Li G, Li F, Ding Q, Kang Q, Huang D, et al: Clinical implication of Keap1 and phosphorylated Nrf2 expression in hepatocellular carcinoma. Cancer Med 5: 2678-2687, 2016

This work is licensed under a Creative Commons Attribution-NonCommercial-NoDerivatives 4.0 International (CC BY-NC-ND 4.0) License. 